\title{
Human Activity Recognition Based on Surrounding Things
}

\author{
Naoharu Yamada, Kenji Sakamoto, Goro Kunito, \\ Kenichi Yamazaki, and Satoshi Tanaka \\ Network Laboratories, NTT DoCoMo, Inc., \\ 3-5 Hikarino-oka, Yokosuka city, Kanagawa 239-8536, Japan \\ \{yamada, sakamoto, kunito, yamazaki, \\ satoshi\}@netlab.nttdocomo.co.jp
}

\begin{abstract}
This paper proposes human activity recognition based on the actual semantics of the human's current location. Since predefining the semantics of location is inadequate to identify human activities, we process information about things to automatically identify the semantics based on the concept of affordance. Ontology is used to deal with the various possible representations of things detected by RFIDs, and a multi-class Naïve Bayesian approach is used to detect multiple actual semantics from the terms representing things. Our approach is suitable for automatically detecting possible activities under a variety of characteristics of things including polysemy and variability. Preliminary experiments on manually collected datasets of things demonstrated its noise tolerance and ability to rapidly detect multiple actual semantics from existing things.
\end{abstract}

\section{Introduction}

Owing to the downsizing and increasing sophistication of computing appliances, the Ubiquitous Computing Environment proposed by Mark Weiser [22] is becoming reality. In the Ubiquitous Computing Environment, people will enjoy new services called "ubiquitous services". The appropriate ubiquitous services are provided depending on user's activities. While traditional services are reactive and uniform for every user, ubiquitous services are proactive and adaptive to each user. For example, when a user is shopping in a food court, the system can tell him what is in his refrigerator and what is missing. When the user unintentionally leaves his umbrella in a shop or train, the system detects the omission and informs the user. One of the essential issues in achieving ubiquitous services is how to recognize human activities since services are provided depending on the user's activities not his explicit requests. If system misjudges the activity, the user will receive useless and annoying services.

This paper presents a method to infer human activities based on the actual semantics of the human's current location. We name it activity space. Activity spaces (AS) are the logically defined spaces in which the user will perform a particular activity. By identifying activity space at user's current position, the system can infer the user's activities. Activity space is characterized by continual creation and disappearance. For example, when a flea market is held at a park, a shopping $A S$ only exists during the period of the flea market. Therefore, we need a way of detecting the existence of 
activity spaces automatically. To solve this issue, we focus on "things" that compose activity spaces. Since things basically have the purpose of existence and affordance [4] that offers people to do with them, they can specify human activities. Each thing can be identified by a Radio Frequency Identification (RFID) tag. Since various terms can be used to represent the same thing, we acquire all representations by utilizing ontology [5]. Activity spaces are detected by employing a topic detection method designed for document handling because we can draw an analogy between documents composed by words and activity spaces composed by things. Preliminary experiments utilizing actual things data demonstrate the feasibility of our proposed method.

\section{Related Work}

Approaches to tackle the essential issue of human activity recognition are classified into two types. One is utilizing wearable sensors [14] and the other is utilizing various sensors attached to things in the environment. Though the former approach is appropriate for fundamental activities such as sitting, standing, and walking by detecting limb motions, it places a burden on users since they wear the devices. For the latter approach, some papers focused on detecting the interaction between humans and objects by utilizing a camera [7] or an ultrasonic sensor [11]. Unfortunately, implementation costs are very high and the approaches only work in the laboratory. Tapia et al. [17] developed environmental state change sensors and Fishkin et al. [3] utilized RFID tags. They recognize user's activities based on sequential data of things that the user touches or grasps. They achieved low-cost implementation and their work are applicable to real world environments. However, it is difficult for them to identify the activities including many non-sequential interactions with various things since the user may perform these activities in various ways.

Our main idea is to focus on activity spaces in identifying human activities. The simple approach is inference based on the predefined semantics of location such as a map [13] [21]. They focused more on how to identify the spatial position of users and less on how to specify the semantics of the spatial position because they assumed that the semantics of a spatial position was static. However, the effectiveness of this approach is limited since the activities that a location can offer are fixed and some locations do not specify just one activity. As for the former, the location semantics can change over time such as a flea market. However, these semantics are not handled at all in prior works. Typical examples of the latter locations are a living room at home or a multipurpose room. Though a living room can support various activities such as studying, working, eating, and playing TV games, the system cannot identify the activities actually supported by a room since it depends on the equipment in the room.

Our approach is to focus on the things forming the user's immediate environment to identify activity spaces. Moreover, things existing in a certain space can be easily detected by RFID tags. They are seen as replacing the barcode in the area of logistics. Some companies or governments now require suppliers to attach RFID tags to every item. EPC Global [2] and Ubiquitous ID center [19] have proposed an ID scheme that makes it possible to put a unique serial number on every item. Considering this background, we can assume that everything will soon have its own RFID tag. This means that RFID tags are the most promising approach to realizing the Ubiquitous Computing Environment. The use of RFID tags demands the use of RFID tag readers. 
They are located in the environment or a user carries one. From the perspective of hardware cost, there is tradeoff between these two methods: if the target space is large and the number of users is small, the former method is better, and otherwise, the latter is better. In this paper, we assume RFID tag readers are placed in the environment.

\section{Activity Space Detection}

This section clarifies the definition, characteristics, and technical issues of an activity space. It then describes the proposed approach based on ontology and multi-class Naïve Bayes for automatic detection of activity spaces.

\subsection{Activity Space: Definition, Characteristics, and Technical Issues}

An activity space is a logically defined space that affords the user some particular activity. Examples are "shopping AS" such as supermarkets, flea markets, and stalls are where users buy commodities. "Eating AS" such as a dining room at home, restaurants, and cafeterias are where we eat and drink. "User's own domains AS" such as the user's own room in their house and the user's desk at the office are where the user keeps his/her possessions. Activity spaces are not simply spaces defined in terms of X-Y-Z coordinates with no regard for semantics; activity spaces are inherently associated with semantics. Activity space is a subconcept of place. With regard to place, Tuan [18] mentioned that "place is space infused with human meaning", and Curry [1] mentioned the several ways in which places are created: naming, categorizing, making a symbol, telling stories, and performing activities. In his categorization, an activity space is a place of performing activities with particular objects.

Activity spaces have the following characteristics.

Dynamics of existence: Activity spaces are dynamically generated, move, and disappear. For example, "a shopping AS" such as a flea market is dynamically generated, moves, and disappears in parks or squares depending on the action of the booth owners. "An eating AS" can be dynamically generated by preparing a meal and disappears after the meal. Each activity space has a different period of existence. Some activity spaces, such as a bedroom in a house, can exist for long periods. On the other hand, the activity spaces such as an eating activity space or a flea market exist for short periods. This characteristic raises a technical issue: the transient activity spaces cannot be identified by using spatial maps.

Spatial relationships: Several Activity Spaces can exist at the same spatial position. For example, while a living room is designed to enable people to get together for meeting or chatting with a family or friends, people do several other activities such as eating and working in a living room. Therefore, there are spatial relationships among activity spaces such as inclusion, overlap, and adjacency. This characteristic raises the fact that multiple activity spaces can occupy the same spatial position.

Therefore, a key technical issue on activity space is multiple activity space detection.

\subsection{Thing-Oriented Activity Spaces Detection and Its Difficulties}

People can generally recognize an activity space simply by "looking at" it. For example, if people look at a kitchen in a house, they can recognize it as a cooking AS. The 
reason is affordance as introduced by Gibson [4]. Affordance is what things offer people to do with them. Affordance enables you to recognize what actions you can do with a thing by just looking at it. For example, a knife offers the function of cutting to people and they can recognize that $a$ knife can be used to cut objects by just looking at the knife. By extending affordance, we believe that a set of things also affords particular activities to people. Therefore, we focus on sets of things to identify activity spaces. However, identifying activity spaces from a set of things suffers from several difficulties. We listed them based on the characteristics of things.

Massiveness: People are surrounded by a huge number of things. While some of them are effective in identifying the activity space, others such as lamp and trash are useless; eliminating the ineffective things is very difficult [P1-1].

Mobility: Things can be moved by several causes. The things that are moved due to the user's intention such as food or dishes for preparing meals are important in identifying the activity space. However, other things that move such as the user's clothing are useless; it is necessary to suppress the noise [P1-2].

Polysemy: Everything has multiple representations. For example, the thing pencil has the meaning of a writing tool and stationery, and at the store, it has the meaning of a commercial goods. Thus, how to represent things is difficult [P2].

Variability: The things that form the same kind of the activity space are different in each activity space. For example, the cooking ASs of different houses have different things. This implies the manual creation of detection rules is extremely difficult [P3]. Furthermore, even if some learning approaches are utilized to automatically extract inference rules, the system cannot deal with unlearned things [P4].

While the above difficulties arise from the characteristics of things, other problems arise from the use of RFIDs: RFID tag detection is not completely reliable because of collision and differences in the interval of ID transmission.

\subsection{Ontology and PMM for Detecting Activity Spaces from Things}

To solve P2 and P4, we utilize ontology that defines explicit formal specifications of terms and the relations among them. As for P1-1, P1-2, P3, and the technical issue of multiple activity spaces detection, we employ the parametric mixture model (PMM) [20], a text classification method, because we draw an analogy between documents composed by words and activity spaces composed by things.

The proposed system consists of four processes: preprocess, represent, learn, and classify. In the preprocess stage, the system aggregates detected RFID tags and extracts distinct things. For example, the system extracts only things that appeared recently to detect newly generated activity spaces. In the represent stage, the system acquires terms that represent each thing. We acquire the attribute information of each detected thing from Physical Markup Language servers (PML servers) [12] of EPC Global. Utilizing the information, all terms representing the things are acquired through ontology. At the learn stage, the probability of a thing being in an activity space is specified by utilizing the terms and supervised activity space data. In the classify stage, the system uses PMM to classify a set of terms into activity spaces. 


\subsubsection{Ontology to Manage Representations}

Ontology has a long history in philosophy as it refers to the subject of existence. One definition of ontology involves the specification of terms in each domain and the relations among them. Ontology sets "basic concept" that represent underlying concept such as pencil and "role concept" that represent the role that a thing plays in a particular domain such as product. In addition, it also sets "is-a relation" to represent the sub concept between two terms. For example, "A pen $i s-a$ writing tool" means $a$ pen is a sub concept of $a$ writing tool [8][9][10]. Utilizing these concepts and relations makes it possible to acquire all terms related to a thing by tracing relations. The lowest terms in each concept are preliminarily linked to the ID of each thing in PML. Among all terms related to a thing, it is necessary to identify the appropriate term for the thing to identify activity spaces. Since it is difficult to identify proper terms and the appropriate level in a hierarchy based on is- $a$ relation, we manually choose the appropriate concept instead of the appropriate terms. For example, basic concept is selected for $a$ working $A S$, and role concept is selected for a shopping AS. This approach, however, leaves unanswered how to select the proper abstraction level in the $i s-a$ relation; this is solved in the next section. To solve $\mathrm{P} 4$, we transform the terms that have not been learned into the terms that have been learned by utilizing is- $a$ relations. For example, if the thing eraser has not been learned but the thing pencil has, we can treat both as stationery, which has already been learned.

\subsubsection{Activity Space Identification Via Topic Detection}

Many schemes for tackling the identification of the topics of documents or web contents have been proposed. The characteristics of their target are very similar to those of our objective: a document consists of a set of words that includes noise such as stop words [15], each document on the same topic consists of different words, but people can identify the topic of a document at a glance. Among the many approaches proposed for topic detection, most assume that a document has only one topic; the parametric mixture model (PMM), however, allows one document to have multiple topics. It employs a probabilistic approach which is efficient and robust against noise; it offers the highest accuracy in detecting multiple topics [20]. Since it is highly likely that multiple activity spaces will be detected from one set of things, we employ PMM.

PMM extends Naïve Bayes [6] to provide multi-topic detection. PMM assumes that a multi-topic document is composed of a mixture of words typical of each topic. Based on this assumption, a multi-topic document can be represented as the linear summation of the word occurrence probability vector of each topic as shown in Eq. (1). Here, $p\left(t_{i} \mid c_{l}\right)$ is calculated using MAP estimation. By replacing (words, topics) with (things, activity spaces), we can use Eq. (1) to detect multiple activity spaces.

$$
\begin{aligned}
& p(d \mid c)=p\left(t_{1}, \ldots, t_{n} \mid c\right)=\prod_{i=1}^{n}\left(\sum_{l=1}^{L} h_{l}(y) p\left(t_{i} \mid c_{l}\right)\right)^{x_{i}} \\
& \text { where } h_{l}(y)=\frac{y_{l}}{\sum_{l=1}^{L} y_{l^{\prime}}}, l=1, \ldots, L, y_{l}=1\left(y_{l} \text { belongs }\right) \text { or } 0\left(y_{l} \text { does not belong }\right) \\
& d: \text { document }, c: \text { topic, } x_{i}: \text { frequency of word } t_{i}, L: \text { No.of topic, } n: \text { No.of word kinds }
\end{aligned}
$$

To select the appropriate abstraction level of an is-a relation, conditional probability p(thing I activity space) is learned utilizing the lists of things at each abstraction level. 
PMM then acquires the classification accuracy of activity spaces though the learned conditional probability. Finally, the abstraction level with the highest classification accuracy is employed to classify a test set of things.

\section{Preliminary Experiment}

Before doing experiments in an actual environment, we did preliminary experiments using actual data that was manually collected. We evaluate the feasibility of the proposed method for activity space detection under the difficulties posed by P1-1, P2, P3, and P4 while P1-2 and the difficulties created by the RFIDs was left for the experiments in actual environments. We did two experiments: exp.1), the detection of frequently changing activity spaces to address $\mathrm{P} 2, \mathrm{P} 3$, and $\mathrm{P} 4$, and exp.2), the detection of an activity space that contains a large number of things to address P1-1. In exp.1, we focused on a table in a living room since it can support several activities as described in 3.1. Since a meeting AS always exists, we detect three activity spaces: just a meeting AS, a meeting AS and a working AS, and a meeting AS and an eating AS. We assume that RFID tag readers are put on the table and detect things on or near the table. Though activity spaces on a table in a living room frequently change, the things on it are relatively few (94 things, 26 kinds). In exp. 2 we focused on a room in a home since each room has many things (836 things, 472 kinds). We detect four types of activity spaces: a living room (a meeting AS), a kitchen (a cooking AS), a bath room (a bathing AS), and a study room (a working AS). We assume that each room has several RFID tag readers. We use F-measure to evaluate the accuracy of activity space detection. The F-measure is defined as the harmonic mean of precision and recall and is widely used in the information retrieval field.

\subsection{Input Data to Detect Activity Spaces}

First of all, we need to acquire the data of real world things that includes actual noise. As for exp.1, we manually identified all things on a table of a typical Japanese home. Although PMM must know of the things of each activity space to learn the conditional probability, a meeting AS always exists when a working AS or a meeting AS exists. Therefore, we eliminate the data of things that indicate just a meeting AS from those of an eating AS or a working AS. Fig. 1 (a) shows the things of each AS. As for

\begin{tabular}{|c|c|}
\hline Activity Space & Things \\
\hline Living place & $\begin{array}{l}1 \text { dining table, } 4 \text { chairs, } 4 \text { cushions, } 4 \text { newspapers, } 1 \text { vase, } 1 \text { jotter, } 5 \text { window } \\
\text { envelops, } 5 \text { ballpoints, } 1 \text { in-basket, } 1 \text { wastepaper basket, } 2 \text { coasters }\end{array}$ \\
\hline Eating place (Breakfast) & 6 dishes, 2 chopsticks, 2 table spoons, 2 mugs, 2 table linens \\
\hline Eating place (Lunch) & 6 dishes, 2 chopsticks, 2 forks, 2 table knives, 2 glasses, 2 table linens \\
\hline Eating place (Dinner) & $\begin{array}{l}6 \text { dishes, } 2 \text { chopsticks, } 2 \text { forks, } 2 \text { table knives, } 2 \text { glasses, } 2 \text { beer cans, } 2 \\
\text { table linens }\end{array}$ \\
\hline Working place & $\begin{array}{l}2 \text { ballpoints, } 4 \text { highlighters, } 1 \text { commonplace book, } 1 \text { digital computer, } 1 \\
\text { power code, } 1 \text { mouse, } 7 \text { files }\end{array}$ \\
\hline
\end{tabular}

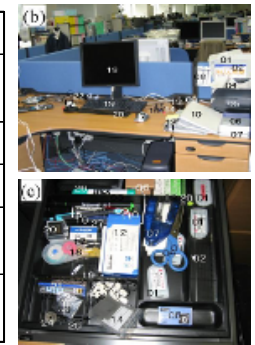

Fig. 1. (a): Things of each activity space for exp.1. (b), (c): Pictures used to identify things in an actual office desk for exp. 2 . 
exp.2, we used the things in an actual Korean family's house as collected by the National Museum of Ethnology [16]. Since the house did not have a study room, we manually identified all things in and on an office desk from photos taken at various angles (Fig. 1 (b) (c))

To represent the data of things in abstract terms, we surveyed existing ontology bases in terms of the number of vocabularies, abstract terms, and hierarchy and the structure of conceptualization. In this survey, we decided to employ WordNet [23]. We set "artifact" in WordNet as abstraction level 1, the most abstract term, and acquired terms on abstraction levels 2 to 6 by utilizing the $i s-a$ relations provided by WordNet. Instead of utilizing PML, we manually set the terms of abstraction level 6 representing each thing. We then added noise to the abstraction data sets with noise ratios of $0 \%, 25 \%$, and $50 \%$. In detail, we added the things of another activity space to reflect the presence of things not related to the activity space. In addition, we randomly eliminated some things from the data sets to reflect RFID detection error and the presence of things without an RFID tag. By randomly adding noise, we created 1000 data sets for each activity space. To include unlearnt things in test data for evaluating P4, we used eating ASs (breakfast) as learning data and those of lunch and dinner as test data in exp.1.

\subsection{Results}

Table 1 shows the F-measure in exp.1. The proposed method successfully detected a meeting AS and an eating AS with a high degree of accuracy, while that of a working AS was not high. This result indicates that a meeting AS and an eating AS have the particular things that clearly identify the activity space while a working AS does not. In detail, while the multiple activity spaces of working and living can be successfully detected, the single activity space of living is classified as the multiple activity spaces of working and living. This is because a meeting AS has some characteristic things of such as ballpoints and jotters. This result also demonstrates the noise tolerance of the proposed method since the accuracy of activity space detection did not drop as the noise ratio was raised. Furthermore, the accuracy of working AS detection increased with the abstraction level. By raising the abstraction level, the number of kinds of terms decreased: 1 kind in level 1 and 34 kinds in level 6. This means that the information amount decreased and the accuracy of activity space detection generally falls. Ontology can provide an explanation: each activity space has many kinds but a few discriminative terms; the use of ontology raised the abstraction level which transformed them into fewer kinds with a larger number of discriminative terms. This demonstrates that ontology can raise the accuracy of activity space detection. Note that it makes sense that the F-measure of level 1 is 0 in most activity spaces since the term of abstraction level 1 is just "Artifact". As for unlearnt things, we did not learn forks, table knives, and glasses. WordNet transformed forks, table knives, and tablespoons into cutlery in level 5. Glasses and mugs, which were known were transformed into container in level 3. Therefore, ontology could utilize unlearnt things for activity space detection by raising the abstraction level.

Table 3 shows the F-measure of each activity space in exp.2; the results also demonstrate the feasibility of the proposed method. Table 2 shows the processing time needed for assessing 4000 sets of things data and the number of kinds of terms in each abstraction level. This demonstrates that the proposed method can rapidly handle 
large sets of things and that increasing the abstraction level makes it possible to reduce the processing time. Furthermore, though 472 kinds of things were aggregated into 17 kinds in abstraction level 2, the F-measure of each activity space did not drop, which obviously demonstrates the effectiveness of ontology.

Table 1. F-measure of detecting each activity space in exp. 1

\begin{tabular}{|c|c|c|c|c|c|c|}
\hline \multicolumn{7}{|c|}{ Meeting AS } \\
\hline noise ratio & Level1 & Level2 & Level3 & Level4 & Level5 & Level6 \\
\hline $0 \%$ & $100.0 \%$ & $100.0 \%$ & $100.0 \%$ & $100.0 \%$ & $100.0 \%$ & $100.0 \%$ \\
\hline $25 \%$ & $100.0 \%$ & $99.9 \%$ & $100.0 \%$ & $100.0 \%$ & $100.0 \%$ & $100.0 \%$ \\
\hline $50 \%$ & $100.0 \%$ & $98.3 \%$ & $100.0 \%$ & $100.0 \%$ & $100.0 \%$ & $100.0 \%$ \\
\hline \multicolumn{7}{|c|}{ Meeting AS and Eating AS } \\
\hline noise ratio & Level1 & Level2 & Level3 & Level4 & Level5 & Level6 \\
\hline $0 \%$ & $0.0 \%$ & $100.0 \%$ & $100.0 \%$ & $100.0 \%$ & $100.0 \%$ & $100.0 \%$ \\
\hline $25 \%$ & $0.0 \%$ & $100.0 \%$ & $100.0 \%$ & $100.0 \%$ & $100.0 \%$ & $100.0 \%$ \\
\hline $50 \%$ & $0.0 \%$ & $100.0 \%$ & $100.0 \%$ & $100.0 \%$ & $99.8 \%$ & $100.0 \%$ \\
\hline \multicolumn{7}{|c|}{ Meeting AS and Working AS } \\
\hline noise ratio & Level1 & Level2 & Level3 & Level4 & Level5 & Level6 \\
\hline $0 \%$ & $0.0 \%$ & $100.0 \%$ & $100.0 \%$ & $100.0 \%$ & $100.0 \%$ & $100.0 \%$ \\
\hline $25 \%$ & $0.0 \%$ & $75.9 \%$ & $93.5 \%$ & $94.2 \%$ & $96.4 \%$ & $90.5 \%$ \\
\hline $50 \%$ & $0.0 \%$ & $62.9 \%$ & $81.0 \%$ & $79.3 \%$ & $86.1 \%$ & $78.0 \%$ \\
\hline
\end{tabular}

Table 2. Processing time for estimating 4000 data sets and the No. of kinds of terms

\begin{tabular}{|l|r|r|r|r|r|r|}
\hline & level1 & level2 & level3 & level4 & level5 & level6 \\
\hline No. of kinds of terms & 1 & 17 & 48 & 149 & 421 & 472 \\
\hline time for estimation (sec) & 37 & 55 & 100 & 227 & 526 & 570 \\
\hline
\end{tabular}

Table 3. F-measure of detecting each activity space in exp. 2

\begin{tabular}{|c|c|c|c|c|c|c|}
\hline noise ratio & Level1 & Level2 & Level3 & Level4 & Level5 & Level6 \\
\hline $0 \%$ & $0 \%$ & $100.0 \%$ & $100.0 \%$ & $100.0 \%$ & $100.0 \%$ & $100.0 \%$ \\
\hline $25 \%$ & $0 \%$ & $97.1 \%$ & $95.6 \%$ & $99.8 \%$ & $98.9 \%$ & $100.0 \%$ \\
\hline $50 \%$ & $0 \%$ & $86.6 \%$ & $92.2 \%$ & $99.4 \%$ & $97.8 \%$ & $100.0 \%$ \\
\hline \multicolumn{7}{|c|}{ Cooking AS } \\
\hline noise ratio & Level1 & Level2 & Level3 & Level4 & Level5 & \begin{tabular}{|l|} 
Level6 \\
\end{tabular} \\
\hline $0 \%$ & $40 \%$ & $100.0 \%$ & $100.0 \%$ & $100.0 \%$ & $100.0 \%$ & $100.0 \%$ \\
\hline $25 \%$ & $40 \%$ & $91.1 \%$ & $96.5 \%$ & $98.7 \%$ & $99.4 \%$ & $100.0 \%$ \\
\hline $50 \%$ & $40 \%$ & $82.5 \%$ & $92.5 \%$ & $97.6 \%$ & $96.9 \%$ & $100.0 \%$ \\
\hline \multicolumn{7}{|c|}{ Meeting AS } \\
\hline noise ratio & Level1 & Level2 & Level3 & Level4 & Level5 & Level6 \\
\hline $0 \%$ & $100 \%$ & $100.0 \%$ & $100.0 \%$ & $100.0 \%$ & $100.0 \%$ & $100.0 \%$ \\
\hline $25 \%$ & $100 \%$ & $94.6 \%$ & $97.2 \%$ & $98.1 \%$ & $98.8 \%$ & $100.0 \%$ \\
\hline $50 \%$ & $100 \%$ & $89.9 \%$ & $94.0 \%$ & $97.5 \%$ & $97.7 \%$ & $100.0 \%$ \\
\hline \multicolumn{7}{|c|}{ Working AS } \\
\hline noise ratio & Level1 & Level2 & Level3 & Level4 & Level5 & Level6 \\
\hline $0 \%$ & $0 \%$ & $100.0 \%$ & $100.0 \%$ & $100.0 \%$ & $100.0 \%$ & $100.0 \%$ \\
\hline $25 \%$ & $0 \%$ & $90.6 \%$ & $97.5 \%$ & $99.3 \%$ & $99.4 \%$ & $100.0 \%$ \\
\hline $50 \%$ & $0 \%$ & $83.9 \%$ & $95.1 \%$ & $98.4 \%$ & $97.4 \%$ & $100.0 \%$ \\
\hline
\end{tabular}

\section{Discussion}

The preliminary experiments described above evaluated the proposed method using actual but manually collected sets of things. Though the findings of these experiments are meaningful and interesting, some problems remain to be evaluated.

Mobility of things: Noise data derived from this characteristic are not included in the manually collected sets. To evaluate it, we need to establish an environment where each thing has an RFID tag and gather the data of RFID tag detection over time.

Human activity inference based on activity spaces: We need to evaluate the accuracy of inferring the user's situation from the activity spaces. To do this, we can compare the activity inferred from activity spaces with actual user activity acquired by asking the user what $\mathrm{s} / \mathrm{he}$ is doing in the environment.

Furthermore, we need to consider the following issues.

Target activity spaces: Activity spaces need to be expanded and refined. As for expansion, we need to acquire as many activity spaces as there are human activities. As for refinement, we need to classify each activity space into more refined activity space. For example, a meeting $A S$ has sub-concepts of a director's meeting $A S$ and a group meeting AS. Ontology would be helpful in achieving this. 
Concepts of ontology: WordNet defines only the basic concepts of terms. Though it is useful to identify many activity spaces, other activity spaces such as selling space are difficult to identify. Since no existing ontology base defines role concept such as product, we need to build the ontology of role concept.

PMM for activity space detection: Since PMM is designed for identifying a topic from a large amount of words, it is difficult to identify the few important things. For example, takeout food consists of few things but important in identifying an eating $A S$. This issue can be solved by adding weight to thing in preprocessing.

Human activity inference in multiple activity spaces: When multiple activity spaces overlap, the system needs to choose some of them to identify user activity. One approach is identifying the activity space generated most recently since a user intentionally moves things to perform a particular action such as preparing meal. Another approach is identifying the thing that the user is interacting with and specifying the activity space with the highest conditional probability.

\section{Conclusion}

This paper proposed a novel approach to recognize human activities based on activity spaces, the spaces that afford humans particular activities. Activity spaces are identified through the things that exist there based on the concept of affordance. We utilize ontology to specify terms representing things and the parametric mixture model to identify activity spaces from the terms. Since activity spaces represent the "actual" semantics of position, activity spaces infer human activities more precisely than conventional approaches based on just location; moreover, this approach is more feasible than those based on just what the user is interacting with. Preliminary experiments demonstrated the noise tolerance, high accuracy of activity space detection, and the ability to rapidly handle large amounts of data. Though we focused on human activities with things, other activities that are independent of things remain to be recognized. Such activities may depend on other entities such as human or time context and in that case, our approach based on ontology and topic detection may be applicable.

\section{References}

1. Curry M.: The Work in the World - Geographical Practice and the Written Word. University of Minnesota Press, ISBN 0-8166-2665-0, 1996.

2. EPC Global: http://www.epcglobalinc.org/

3. Fishkin K., Jiang B., Philipose M., Roy S.: I Sense a Disturbance in the Force: Unobtrusive Detection of Interactions with RFID-tagged Objects. Proc of 6th Intl. Conference on Ubiquitous Computing (UbiComp2004), pp.268-282, 2004.

4. Gibson J.: The Ecological Approach to Visual Perception. Lawrence Erlbaum Assoc Inc, ISBN: 0898599598, 1979.

5. Gruber T.: A translation approach to portable ontologies. Knowledge Acquisition, 5(2):199-220, 1993.

6. McCallum A., Nigam K.: A Comparison of Event Models for Naïve Bayes Text Classification. Proc. of Intl. Workshop on Learning for Text Categorization in AAAI-98, 1998. 
7. Moore D., Essa I., Hayes M.: Exploiting Human Actions and Object Context for Recognition Tasks. Proc. of 4th Intl. Conference on Computer Vision (ICCV'99), 1999.

8. Mizoguchi R.: Tutorial on ontological engineering Part1: Introduction to Ontological Engineering, New Generation Computing, OhmSha\&Springer, Vol.21, No.4, pp.365-384, 2003.

9. Mizoguchi R.: Tutorial on ontological engineering Part2: Ontology development, tools and languages, New Generation Computing, OhmSha\&Springer, Vol.22, No.1, pp.61-96, 2004.

10. Mizoguchi R.: Tutorial on ontological engineering Part3: Advanced course of ontological engineering, New Generation Computing, OhmSha\&Springer, Vol.22, No.2, 2004.

11. Nishida Y., Kitamura K., Hori T., Nishitani A., Kanade T., Mizoguchi H.: Quick Realization of Function for Detecting Human Activity Events by Ultrasonic 3D Tag and Stereo Vision. Proc. of 2nd IEEE Intl. Conference on Pervasive Computing and Communications (PerCom2004), pp. 43-54, 2004.

12. PML Core Specification 1.0: http://www.epcglobalinc.org/standards_technology/Secure/ v1.0/PML_Core_Specification_v1.0.pdf

13. Schilit B., Adams N., Gold R., Tso M., Want R.: ParcTab Mobile Computing System. Proc. of 4th Workshop on Workstation Operating Systems (WWOS-IV), pp.34-39, 1993.

14. Seon-Woo L., Mase K.: Activity and Location Recognition Using Wearable Sensors. Pervasive Computing, pp.10-18, Sep.2002.

15. Stop list: ftp://ftp.cs.cornell.edu/pub/smart/english.stop

16. The National Museum of Ethnology: Seoul Style 2002. ISBN: 4915696465, 2002.

17. Tapia E., Intille S., Larson K.: Activity Recognition in the Home Using Simple and Ubiquitous Sensors. Proc. of 2nd Intl. Conference on Pervasive Computing 2004 (Pervasive2004), pp.158-175, 2004.

18. Tuan Y.: Space and Place: The Perspective of Experience. ISBN: 0816638772, 1977.

19. Ubiquitous ID Center: http://www.uidcenter.org/

20. Ueda N., Saito K.: Singleshot detection of multi-category text using parametric mixture models. Proc. of 8th Intl. Conference on Knowledge Discovery and Data Mining (SIGKDD2002), pp. 626-631, 2002.

21. Ward A., Jones A., Hopper A.: A New Location Technique for the Active Office. IEEE Personal Communications, Vol. 4, No. 5, pp.42-47, 1997.

22. Weiser M.: The Computer for the 21st century. Scientific American, pp.94-104, Sep.1991.

23. WordNet: http://wordnet.princeton.edu/ 